Canad. Math. Bull. Vol. 22 (2), 1979

\title{
RINGS ALL OF WHOSE PIERCE STALKS ARE LOCAL
}

\author{
BY \\ W. D. BURGESS* AND W. STEPHENSON
}

The aim of this paper is to give a number of characterizations of the rings of the title. In particular, these turn out to be precisely those exchange rings whose idempotents are all central. They are also those rings in which every element is the sum of a unit and a central idempotent.

1. Preliminaries. The reader is referred to [3] or [4] for undefined ring terminology. In addition, familiarity with the basic properties of the Pierce sheaf is assumed (see, for example [8] or [1] for details). For a ring $R$ (always with 1) we let $X=\operatorname{Spec} \mathbf{B}(R)$ where $\mathbf{B}(R)$ is the Boolean algebra of central idempotents of $R$. For $x \in X$, the Pierce stalk at $x$ is denoted by $R_{x}=R / R x$ and the image of $r \in R$ in $R_{x}$ by $r_{x}$. Throughout a local ring means a ring with a unique maximal left ideal.

In [2] a decomposition of any ring $R$ as a subdirect product of its maximal indecomposable factors (mi-factors) was introduced. This was achieved by iterating the Pierce sheaf construction to obtain a set of ideals $M$ where the ideals $I \in \mathcal{M}$ are minimal with respect to the property that $R / I$ is indecomposable (i.e., without non-trivial central idempotents). Then $R$ is a subdirect product of the $R / I=R_{I}, I \in M$ and many properties "lift" from the $R_{I}$ to $R$. In case the Pierce stalks of $R$ are indecomposable, $M=\{R x \mid x \in X\}$.

Proposition 1.1. The following are equivalent for a ring $R$.

(i) Every idempotent of $R$ is central.

(ii) For each $x \in X, R_{x}$ has no non-trivial idempotents.

(iii) For each $I \in M, R_{I}$ has no non-trivial idempotents.

When these conditions are satisfied, the Pierce stalks and the mi-factors coincide.

Proof. (i) $\Rightarrow$ (ii). If $r_{x}^{2}=r_{x}$ for some $r \in R, x \in X$, then $\left(r^{2}-r\right) e=0$ for some $e \in \mathbf{B}(R)$, $e \notin x$. Hence $(r e)^{2}=r e$ and so $r e \in \mathbf{B}(R)$. But $r_{x}=r_{x} e_{x}=(r e)_{x}=0_{x}$ or $1_{x}$. (ii) $\Rightarrow$ (iii). Since the Pierce stalks are indecomposable they coincide with the mi-factors. (iii) $\Rightarrow$ (i). If $e$ is an idempotent of $R$ then $e_{I}$ is 0 or 1 in each $R_{I}$, $I \in M$. Hence $e$ is a central idempotent since $R$ is a subdirect product of the $R_{I}$.

\footnotetext{
* The first author acknowledges the support of grant A-7539 of the National Research Council of Canada.

Received by the editors March 29, 1978 and, in revised form, August 13, 1978.
} 
Proposition 1.2. The following are equivalent for a ring $R$.

(i) Every $r \in R$ can be written $r=u+e$ where $u$ is a unit and $e \in \mathbf{B}(R)$.

(ii) $R_{x}$ is a local ring for all $x \in X$.

(iii) $R_{I}$ is a local ring for all $I \in M$.

Proof. (ii) $\Leftrightarrow$ (iii). This follows from 1.1. (i) $\Rightarrow$ (ii). Suppose that $r=u+e, u$ a unit and $e \in \mathbf{B}(R)$. Then for $x \in X, r_{x}=u_{x}+e_{x}$. But $e_{x}=0$ or 1 so that $r_{x}$ is a unit or $1-r_{x}$ is a unit.

(ii) $\Rightarrow$ (i). Let $r \in R$. For $x \in X, r_{x}$ or $1-r_{x}$ is a unit so there exists a clopen set (closed and open) in $X, U_{x}$ with $x \in U_{x}$, such that for all $y \in U_{x}, r_{y}$ is a unit or for all $y \in U_{x}, 1-r_{y}$ is a unit. Using the standard tool of Pierce sheaves, there exist disjoint clopen sets $C$ and $D$ with $C \cup D=X$ such that $r_{x}$ is a unit for all $x \in C$ and $1-r_{x}$ is a unit for $x \in D$. Let $e \in \mathbf{B}(R)$ be such that $e_{x}=1$ for all $x \in D$ and $e_{x}=0$ for all $x \in C$. Then $r-e$ is a unit giving the result.

EXAMPLES. Any direct product of local rings satisfies the conditions of 1.2 as does $\mathbf{C}(Y, L)$, the ring of continuous functions from a topological space $Y$ to a discrete local ring $L$.

Remark. If we denote by $\operatorname{Max}(R)$ the set of maximal left ideals of $R$ then $\alpha: \operatorname{Max}(R) \rightarrow X$, given by $\alpha(M)=M \cup \mathbf{B}(R)$, is a surjection. This mapping is a bijection if and only if $R$ satisfies the conditions of 1.2.

2. Some general considerations. In this section we develop some tools in order to study the rings of 1.2 in more detail. For a ring $R$ the nil and Jacobson radicals are denoted by $\mathbf{N}(R)$ and $\mathbf{J}(R)$, respectively.

Let $\mathscr{C}$ be a property of rings. A ring is said to be homomorphically a $\mathscr{C}$-ring (an $H \mathscr{C}$-ring for short) if every homomorphic image of $R$, including $R$, is a $\mathscr{C}$-ring. We note that Levitzki used the terminology "faithful $\mathscr{C}$-ring" in a number of particular cases (see [5]).

Recall that a ring $R$ is (left) fully idempotent if $I^{2}=I$ for every (left) ideal of $R$. It is readily seen that $R$ is fully idempotent if and only if it is a homomorphically semiprime ring.

LEMMA 2.1. For a ring $R, R$ left fully idempotent implies $R$ homomorphically semiprimitive which implies $R$ fully idempotent.

Proof. The second implication follows from the above remarks. Suppose that $R$ is left fully idempotent and $r \in \mathbf{J}(R)$. Then $R r R r=R r$ giving $r=\Sigma x_{i} r y_{i} r$ for some $x_{i}, y_{i} \in R$. Hence $\left(1-\Sigma x_{i} r y_{i}\right) r=0$ which implies $r=0$. The property of being left fully idempotent is preserved by homomorphic images giving the result.

REMARK. It can be shown that neither of the converses of the above lemma hold in general. 
We now consider the relationships between $\mathbf{J}(R), \mathbf{J}\left(R_{x}\right), x \in X$ and $\mathbf{J}\left(R_{I}\right)$, $I \in \mathcal{M}$. The inverse images of $\mathbf{J}\left(R_{x}\right)$ and $\mathbf{J}\left(R_{I}\right)$ in $R$ are denoted by $\mathbf{J}^{\prime}\left(R_{x}\right)$ and $\mathbf{J}^{\prime}\left(R_{I}\right)$, respectively. We have that $\mathbf{J}^{\prime}\left(R_{x}\right)=\bigcap\{P \mid P \supseteq R x$ and $P$ is a left primitive ideal of $R\}$ and $\mathbf{J}^{\prime}\left(R_{I}\right)=\bigcap\{P \mid P \supseteq I$ and $P$ is a left primitive ideal of $R\}$. For any $I \in \mathcal{M}$ there exists a unique $x \in X$ with $x \subseteq I([2,1.4])$ so that $\mathbf{J}(R) \subseteq \bigcap$ $\left\{\mathbf{J}^{\prime}\left(\boldsymbol{R}_{x}\right) \mid x \in X\right\} \subseteq \bigcap\left\{\mathbf{J}^{\prime}\left(\boldsymbol{R}_{I}\right) \mid I \in \mathcal{M}\right\}$. However for any left primitive ideal $\boldsymbol{P}$ of $\boldsymbol{R}$ there exist $I \in \mathcal{M}$ such that $I \subseteq P([2,1.7])$. Hence,

Proposition 2.2. For any ring $R, \mathbf{J}(R)=\bigcap\left\{\mathbf{J}^{\prime}\left(R_{x}\right) \mid x \in I\right\}=\bigcap\left\{\mathbf{J}^{\prime}\left(R_{I}\right) \mid I \in M\right\}$. In particular $R / \mathbf{J}(R)$ is a subdirect product of the rings $\left\{R_{x} / \mathbf{J}\left(R_{x}\right) \mid x \in X\right\}$ and of $\left\{R_{I} / \mathbf{J}\left(R_{I}\right) \mid I \in M\right\}$.

We always have that $\mathbf{J}(R)_{x} \subseteq \mathbf{J}\left(R_{x}\right)$ and $\mathbf{J}(R)_{I} \subseteq \mathbf{J}\left(R_{I}\right)$ but equality, that is, $\mathbf{J}\left(R_{x}\right)=\mathbf{J}(R)+R x$ or $\mathbf{J}\left(R_{I}\right)=\mathbf{J}(R)+I$, need not hold in general. We next investigate a case where it does hold.

Propostrion 2.3. Let $\mathscr{A}$ be a set of ideals of a ring $R$ such that $I=$ $\bigcap\{I+K \mid K \in \mathscr{A}\}$ for every ideal I such that $\mathbf{J}(R) \subseteq I$. Then putting $R / K=R_{K}$, the following are equivalent

(i) $R / \mathbf{J}(R)$ is homomorphically semiprimitive.

(ii) $R_{K} / \mathbf{J}\left(R_{K}\right)$ is homomorphically semiprimitive and $\mathbf{J}(R)_{K}=\mathbf{J}\left(R_{K}\right)$ for all $K \in \mathscr{A}$.

Proof. Assume (i) then, since $R_{K} / \mathbf{J}\left(R_{K}\right)$ is a homomorphic image of $R / \mathbf{J}(R)$, it is homomorphically semiprimitive. Further, $\mathbf{J}(R)+K \subseteq \mathbf{J}\left(R_{K}\right)$ and since $R / \mathbf{J}(R)+K$ is semiprimitive, equality follows.

Now assume (ii). Let $I$ be an ideal containing $J(R)$. Then $I=$ $\bigcap\{I+K \mid K \in \mathscr{A}\}$. Since $R / I+K$ is semiprimitive for $K \in \mathscr{A}$, it follows that $R / I$ is semiprimitive.

REMARK. By 1.7 of [8] and 1.9 of [2], 2.3 applies to $\mathscr{A}=\{R x \mid x \in X\}$ and to $\mathscr{A}=\mathcal{M}$.

Corollary 2.4. The following are equivalent for a ring $R$. (i) $R$ is homomorphically semiprimitive, (ii) $R_{x}$ is homomorphically semiprimitive for all $x \in X$. (iii) $R_{I}$ is homomorphically semiprimitive for all $I \in M$.

3. Further characterizations. Firstly recall that a ring $R$ is reduced if it contains no non-zero nilpotent elements. Secondly, a ring is an I-ring (see [3, p. 210]) if every non-nil left ideal contains a non-zero idempotent. We generalize this and call a ring $R$ a $J I$-ring if every left ideal not contained in $\mathbf{J}(R)$ contains a non-zero idempotent. As for $I$-rings it is easy to show that the definition of a $J I$-ring is, in fact, left-right symmetric. It is also immediate that $R$ is an $I$-ring if and only if it is a $J I$-ring such that $\mathbf{N}(R)=\mathbf{J}(R)$. We shall 
consider $H I$ and $H J I$-rings (recall the definition preceding 2.1). In [5], $H I$-rings were called $F I$-rings.

ExAMPLE. If $R / \mathbf{J}(R)$ is regular and $\mathbf{J}(R)$ is nil then $R$ is an HJI-ring.

THEOREM 3.1. The following are equivalent for a ring $R$.

(i) $R$ satisfies the conditions of 1.2 .

(ii) (a) Every idempotent is central. (b) Every maximal left ideal is two-sided. (c) Idempotents can be lifted modulo every ideal.

(iii) (a) Every idempotent is central. (b) $R$ is an HJI-ring. Further, in such a ring, $R / \mathbf{J}(R)$ is reduced, $\mathbf{B}(R)=\mathbf{B}(R / \mathbf{J}(R))$ and identifying $\operatorname{Spec} \mathbf{B}(R / \mathbf{J}(R))$ with $X,(R / \mathbf{J}(R))_{x}=R /(\mathbf{J}(R)+R x)$.

Proof. (i) $\Rightarrow$ (ii). Parts (a) and (b) follow directly from the fact that the $R_{x}$, $x \in X$, are local. Now suppose that $a^{2}-a \in I$ for some $a \in R$ and ideal $I$. Now $a=u+e$ where $e \in \mathbf{B}(R)$ and $u$ is a unit (by 1.2). Then $a^{2}-a=$ $(u+e)^{2}-(u+e)=u(u+2 e-1) \in I$. Therefore $u+2 e-1 \in I$ and so $a=u+e=$ $1-e+(u+2 e-1)$ giving that $a+I$ lifts to $1-e$ modulo $I$.

(ii) $\Rightarrow$ (iii). Let $I$ be an ideal maximal with respect to the property that it contain no idempotents other than 0 . If $I \neq \mathbf{J}(R)$ there is a maximal left ideal $M$, which is an ideal by (ii) (b), with $I \notin M$. Then $R / I \cap M \cong R / I \times R / M$. Lift the idempotent corresponding to $(0,1)$ modulo $I \cap M$ to an idempotent $e \in R$. Then $e \neq 0$ and $e \in I$. This contradiction shows that $I=\mathbf{J}(R)$.

(iii) $\Rightarrow$ (i). Since the idempotents of $R$ are all central, the Pierce stalks of $\boldsymbol{R}$ are indecomposable $J I$-rings. Such rings are local.

Finally if $R$ satisfies the conditions (i), (ii) and (iii), $R / \mathbf{J}(R)$ is a subdirect product of the diversion rings $R_{x} / \mathbf{J}\left(R_{x}\right)$ by 2.2. By (ii) (c), the map $\alpha: \mathbf{B}(R) \rightarrow$ $\mathbf{B}(R / J(R))$ defined by $\alpha(e)=e+\mathbf{J}(R)$ is onto. Suppose for $e, f \in \mathbf{B}(R)$ that $e+\mathbf{J}(R)=f+\mathbf{J}(R)$. Then the idempotents $e-f e$ and $f-f e$ are in $\mathbf{J}(R)$. Hence $e=e f=f e=f$, so that $\alpha$ is a bijection. Using $\alpha$ to identify $\mathbf{B}(R)$ with $\mathbf{b}(R / \mathbf{J}(R))$ we see that $(R / \mathbf{J}(R))_{x}=(R / \mathbf{J}(R)) /(\mathbf{J}(R)+R x / \mathbf{J}(R))=R /(\mathbf{J}(R)+R x)$.

EXAMPLES. (i) The conditions in 3.1 (ii) cannot be weakened. That is, there are rings satisfying two of (a), (b) and (c) but not the third. The ring of upper triangular $2 \times 2$ matrices over a field satisfies (b) and (c), but not (a). The ring $\mathbf{Z}$ satisfies (a) and (b) but not (c). A simple domain which is not a division ring satisfies (a) and (c) but not (b).

(ii) Looking at 3.1 might suggest that rings $R$ satisfying the conditions of 3.1 would be such that $R / \mathbf{J}(R)$ is strongly regular. This is not the case. Let $S$ be a commutative local domain and $Q$ its field of quotients and suppose $Q \neq S$. As in [1, Corollary 3], a ring $R$ can be constructed so that $X=\operatorname{Spec} \mathbf{B}(R)$ is $\mathbf{N} \cup\{\infty\}$, the one-point compactification of the discrete space $\mathbf{N}$ and such that the stalks at the discrete points are $Q$ and at $\infty$ the stalk of $R$ is $S$. ( $R$ is the 
ring of sequences from $Q$ which are eventually constant with the constant value in $S$.) It is easily shown that $R$ is a commutative ring in which every non-zero ideal contains a non-zero idempotent. Hence $\mathbf{J}(R)=0$ but $R$ is not regular. However the ring $T$ constructed similarly with all stalks $S$ (the ring of all eventually constant sequences from $S$ ) is such that $T / \mathbf{J}(T)$ is the regular ring of all eventually constant sequences from $S / J(S)$. The situation is clarified by the following.

Propostrion 3.2. Let $R$ be a ring whose Pierce stalks are local. Then the following are equivalent.

(i) $R / \mathbf{J}(R)$ is strongly regular.

(ii) $R / \mathbf{J}(R)$ is biregular.

(iii) $R / \mathbf{J}(R)$ is homomorphically semiprimitive.

Proof. (i) $\Rightarrow$ (ii) and (ii) $\Rightarrow$ (iii) are obvious. To prove (iii) $\Rightarrow$ (i) we have, by 2.3, that $\mathbf{J}(R)_{x}=\mathbf{J}(R)+R x$. By 3.1, $(R / \mathbf{J}(R))_{x}=R / \mathbf{J}(R)+R x=R / \mathbf{J}^{\prime}\left(R_{x}\right)=$ $\boldsymbol{R}_{x} / \mathbf{J}\left(\boldsymbol{R}_{x}\right)$ which is a division ring. A ring whose stalks are division rings is strongly regular.

A ring $R$ is said to be left $\pi$-regular if for $a \in R$ there is $x \in R, n \in \mathbf{N}$, such that $a^{n}=x a^{n+1}$. Left $\pi$-regular rings are $H I$-rings. Note also that a local ring $R$ is left $\pi$-regular if and only if $\mathbf{N}(R)=\mathbf{J}(R)$ if and only if $R$ is an $I$-ring.

Proposition 3.3. The following are equivalent for a ring $R$.

(i) $R$ is an HI-ring all of whose idempotents are central.

(ii) $R$ is a left $\pi$-regular ring all of whose idempotents are central.

(iii) $R_{x}$ is a local left $\pi$-regular ring for all $x \in X$.

Proof. (i) $\Rightarrow$ (iii). This follows from 1.1 which shows that the stalks of $R$ are I-rings with no non-trivial idempotents. Such rings are local. (iii) $\Rightarrow$ (ii). This follows from 1.1 and the fact that "left $\pi$-regular" lifts from the stalks to $R$ be the standard Pierce sheaf argument. (ii) $\Rightarrow$ (i). This is obvious.

A reduced local $I$-ring is a division ring, which implies the following remark. A ring $R$ is a reduced $H I$-ring if and only if $R$ is a reduced left $\pi$-regular ring if and only if $R$ is strongly regular.

Finally we observe the connections between the class of rings whose Pierce stalks are local and a class of rings, called exchange rings, studied by Monk in [6]. The original definition need not concern us here but exchange rings are characterized by the following: $R$ is an exchange ring if given $a \in R$ there exist $b, c \in R$ such that $b=b a b$ and $c(1-a)(1-b a)=1-b a$. It can be seen that this is equivalent to: If $I$ and $J$ are left ideals of $R$ such that $I+J=R$, then there exists an idempotent $e \in I$ such that $R e+J=R$. (private communication from Goodearl to Monk). For any left ideal $I$ not contained in $\mathbf{J}(R)$ there is a left ideal $J \neq R$ such that $I+J=R$. Hence we see that an exchange ring is an HJI-ring. 
The exact connection between HJI-rings and exchange rings is not clear. Our next result generalizes from the commutative case Theorem 3 of [6].

THEOREM 3.4. The following are equivalent for a ring $R$.

(i) The Pierce stalks of $R$ are local.

(ii) $R$ is an exchange ring all of whose indempotents are central.

Proof. (ii) $\Rightarrow$ (i). This follows from 3.1 (iii) since an exchange ring is an $H J I$-ring. (i) $\Rightarrow$ (ii). This follows since any local ring is an exchange ring and the characterization of exchange rings in terms of elements is one which lifts from the Pierce stalks to the ring by the "standard" Pierce sheaf argument.

If $R$ is a ring all of whose idempotents are central then the equivalence of " $R$ is an exchange ring" and "every element is the sum of a unit and an idempotent" was proved by Nicholson in [7, Proposition 1.8] using quite different techniques. However as we have seen the assumption that idempotents be central is only necessary in the first statement.

\section{REFERENCES}

1. W. D. Burgess and W. Stephenson, Pierce sheaves of noncommutative rings, Comm. in Algebra, 4 (1976), 51-75.

2. W. D. Burgess and W. Stephenson, An analogue of the Pierce sheaf for non-commutative rings, Comm. in Algebra, 6 (1978), 863-886.

3. N. Jacobson, Structure of Rings, Amer. Math. Soc. Colloquium Publications, 37, Providence, R.I., 1964.

4. J. Lambek, Lectures on Rings and Modules, Blaisdell, Waltham, Mass., 1966.

5. J. Levitzki, On the structure of algebraic algebras and related rings, Trans. Amer. Math. Soc., 74 (1953), 384-409.

6. G. S. Monk, A characterization of exchange rings, Proc. Amer. Math. Soc., 35 (1972), 349-353.

7. W. K. Nicholson, Lifting idempotents and exchange rings, Trans. Amer. Math. Soc., 229 (1977), 269-278.

8. R. S. Pierce, Modules over commutative regular rings, Memoires Amer. Math. Soc., 70 (1967).

DEPARTMENT OF MATHEMATICS

FACULTY OF SCIENCE AND ENGINEERING

UNIVERSTTY OF OTTAWA

OTtAWA, CANADA

K1N 6N5

BedFord College

REGENT'S PARK

LONDON NW1 4NS

U.K. 